\title{
Education and Social Capital - Corrigendum
}

\author{
Brendan Apfeld, Emanuel Coman, John Gerring and Stephen Jessee
}

DOI: https://doi.org/10.1017/XPS.2021.6; Published online by Cambridge University Press 10 March 2021

In the original publication of Apfeld et al (2021), a coding mistake caused the results plotted in Figure 4, which separately estimates the effect of university attendance on social capital by respondents' year of high school graduation, to be incorrect. The corrected figure appears below.

The corrected results, while somewhat different, do not change the overall inconclusive nature of these findings. As stated in the original article, it would appear that once the data are broken into subsamples there is inadequate power to estimate each subgroup effect precisely enough to learn which are larger and which are smaller. Note that the point estimates are not generally centered inside the confidence intervals because the confidence intervals use a robust bias correction while the point estimates do not, both following the standard options in the function used for these analyses (see original paper for further description).

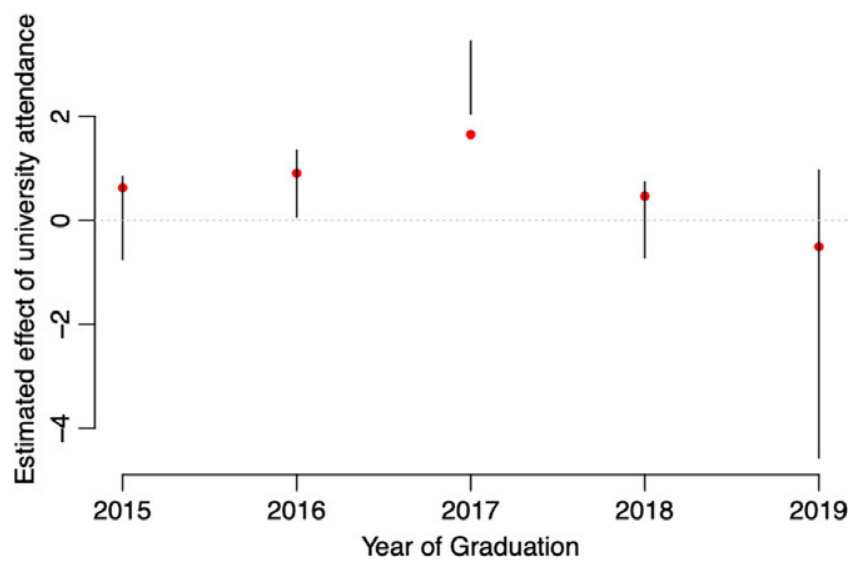

Figure 4

Effect of University Attendance on Social Capital by Year of HS Graduation.

Points show estimated treatment effect (with vertical bars showing robust $95 \%$ confidence intervals) using only respondents who graduated in the specified year, analyzed in the same way as the main results in Table 1. 
The change to these subsidiary results from Figure 4 does not impact the authors' main findings or any of the other results in the article outside of this particular figure.

\section{Reference}

Apfeld, Brendan, Emanuel Coman, John Gerring, and Stephen Jessee. "Education and Social Capital." Journal of Experimental Political Science, first published online 10 March 2021. doi: 10.1017/XPS. 2021.6.

Cite this article: Apfeld B, Coman E, Gerring J, and Jessee S (2022). Education and Social Capital Corrigendum. Journal of Experimental Political Science 9, 278-279. https://doi.org/10.1017/XPS.2021.14 